\title{
Generalized Nonadditive Entropies and Quantum Entanglement
}

\author{
N. Canosa and R. Rossignoli \\ Departamento de Física, Universidad Nacional de La Plata, C.C. 67, 1900 La Plata, Argentina
}

(Received 22 October 2001; published 10 April 2002)

\begin{abstract}
We examine the inference of quantum density operators from incomplete information by means of the maximization of general nonadditive entropic forms. Extended thermodynamic relations are given. When applied to a bipartite spin $\frac{1}{2}$ system, the formalism allows one to avoid fake entanglement for data based on the Bell-Clauser-Horne-Shimony-Holt observable, and, in general, on any set of Bell constraints. Particular results obtained with the Tsallis entropy and with an introduced exponential entropic form are also discussed.
\end{abstract}

DOI: 10.1103/PhysRevLett.88.170401

PACS numbers: 03.65.Ud, 05.30.-d, 03.67.-a

The relation between two fundamental concepts, entropy and quantum entanglement, has recently aroused great interest in quantum information theory [1-8]. A system composed of two subsystems, $A$ and $B$, is said to be $u n$ entangled or separable, if and only if the density operator $\rho$ can be written as a convex combination of uncorrelated densities, i.e., $\rho=\sum_{j} q_{j} \rho_{j}^{A} \otimes \rho_{j}^{B}$, with $q_{j} \geq 0$. Otherwise, the system is said to be entangled or inseparable, in which case it may not admit a local description in terms of hidden variables. The system becomes then suitable, in principle, for applications such as quantum cryptography [9] and teleportation [10].

When the available information about the system is incomplete, consisting, for instance, of the expectation values of a reduced set of observables, one faces the problem of first determining if entanglement is actually implied by the data, and then selecting the most probable or representative density operator compatible with these data. An ideal inference scheme in this scenario should then (a) avoid fake entanglement [1], i.e., should not yield an entangled density if there is a separable density that reproduces the data, (b) be least biased, in the sense that some measure of lack of information is maximized, and (c) be simple enough to be readily applied. As shown in [1], the standard approach based on the direct maximization of the von Neumann entropy, $S=-\operatorname{Tr} \rho \ln \rho$, does not comply with (a) already for two spin $\frac{1}{2}$ systems. The essential reason is that this entropy is not a good entanglement indicator $[5,7,8]$, even in those cases where entanglement is fully determined by the eigenvalues of $\rho$. A solution was also provided in [1]: One should first determine the set of densities that minimize entanglement, and then maximize entropy within this set. Although certainly rigorous, this procedure is not easy to implement in general, and departs conceptually from a more basic approach based on the maximization of a single information measure.

As is well known, the von Neumann entropy is based on the Shannon information measure, which is the unique one satisfying the four Khinchin axioms [11]. However, if the fourth axiom, which is concerned with additivity, is lifted, other information measures become feasible. The most famous recent example is the Tsallis entropy [12], which has been applied to a wide range of phenomena characterized by nonextensivity [13], including recently the problem of quantum entanglement $[3,4]$.

The aim of this work is first to discuss more general nonadditive entropic forms, based on arbitrary concave functions, and the ensuing densities that maximize these forms subject to given constraints. Though sharing many features with the usual von Neumann based statistics, the extended formalism opens new possibilities, in particular that of approaching, for certain functions, densities whose largest eigenvalue has the minimum value compatible with the available data. For a system of two qubits, this allows one to satisfy previous requirements (a), (b), and (c) simultaneously for any set of Bell constraints, by means of a single maximization. In particular, for information based on the Bell-Clauser-Horne-Shimony-Holt (CHSH) observable [1], we will show that fake entanglement can be averted even without including data about the dispersion (in contrast with $[2,3]$ ), for a wide class of entropic functions. As particular cases, we will examine results obtained with the Tsallis entropy, applied here with standard expectation values (as opposed to [3], where the $q$-expectation values were used), and will also introduce an exponential entropic function, which will provide fully analytic results.

Given a density operator $\rho=\sum_{i} p_{i}|i\rangle\langle i|$, with $p_{i} \geq 0$, $\operatorname{Tr} \rho=\sum_{i} p_{i}=1$, and the sum running over a complete set of orthonormal states $\left(\sum_{i}|i\rangle\langle i|=I\right)$, we will consider the entropic form,

$$
S_{f}(\rho) \equiv \operatorname{Tr} f(\rho)=\sum_{i} f\left(p_{i}\right),
$$

where $f$ is a smooth concave function $\left[f^{\prime \prime}(p)<0\right.$ for $p \in(0,1)$ and $f(p)$ continuous for $p \in[0,1]]$ satisfying $f(0)=f(1)=0$. Although Eq. (1) is certainly not the most general information measure, it is the most simple generalization of the von Neumann entropy $[f(p)=-p \ln p]$ and comprises useful extensions. With the exception of additivity, the basic properties of entropy are satisfied for an arbitrary function $f$ of this form. In particular, $S_{f}(\rho) \geq 0$, with $S_{f}(\rho)=0$ only in the case of a pure state $\left(\rho^{2}=\rho\right)$, its maximum is attained 
for a uniform distribution $\left(p_{i}=1 / n\right.$, with $n$ the space dimension), with the maximum value $n f(1 / n)$ increasing with $n$, and it is not affected by states with $p_{i}=$ 0 . Concavity of $f$ implies concavity of $S_{f}(\rho)$ [14], i.e., $S_{f}\left(\sum_{j} q_{j} \rho_{j}\right) \geq \sum_{j} q_{j} S_{f}\left(\rho_{j}\right)$, with $q_{j} \geq 0, \sum_{j} q_{j}=1$, as well as the important property that $S_{f}(\rho)$ cannot decrease by dephasing, i.e., by removing the off-diagonal elements of $\rho$ in any basis of orthonormal states $\left|i_{o}\right\rangle$ :

$$
S_{f}(\rho) \leq S_{f}\left(\rho_{o}\right), \quad \rho_{o}=\sum_{i}\left\langle i_{o}|\rho| i_{o}\right\rangle\left|i_{o}\right\rangle\left\langle i_{o}\right| .
$$

A sufficient condition for $S_{f}$ to be sub(super)additive is that $p f^{\prime \prime}(p)$ be a decreasing (increasing) function of $p$ for $p \in(0,1)$, since in this case $f(p q)-q f(p)-p f(q) \leq$ $0(\geq 0) \forall p, q \in[0,1]$, implying [15]

$$
\begin{gathered}
S_{f}\left(\rho^{A} \otimes \rho^{B}\right) \leq S_{f}\left(\rho^{A}\right)+S_{f}\left(\rho^{B}\right) \\
\quad \text { if }\left[p f^{\prime \prime}(p)\right]^{\prime} \geq 0 .
\end{gathered}
$$

Additivity among entropies of the form (1) holds only if $\left[p f^{\prime \prime}(p)\right]^{\prime}=0$, which leads immediately to the Shannon form $f(p)=-k p \ln p, k>0$.

Maximization of $S_{f}(\rho)$ subject to the constraints of $m+1$ expectation values $\left\langle O_{\alpha}\right\rangle=\operatorname{Tr} \rho O_{\alpha}, \alpha=0, \ldots, m$, where $O_{\alpha}$ are linearly independent observables (not necessarily commuting) and we have set $O_{0}=I$ to account for normalization $(\langle I\rangle=1)$, leads to the maximization of

$$
\bar{S}_{f}(\rho)=S_{f}(\rho)-\sum_{\alpha} \lambda_{\alpha}\left\langle O_{\alpha}\right\rangle=\operatorname{Tr}[f(\rho)-\rho h],
$$

where $h=\sum_{\alpha} \lambda_{\alpha} O_{\alpha}$ and $\lambda_{\alpha}$ are Lagrange multipliers. Writing $h=\sum_{i} h_{i}\left|i_{h}\right\rangle\left\langle i_{h}\right|$, the maximum is attained for

$$
\begin{gathered}
\rho=p(h)=\sum_{i} p_{i}\left|i_{h}\right\rangle\left\langle i_{h}\right|, \\
p_{i}=p\left(h_{i}\right) \equiv \begin{cases}{\left[f^{\prime}\right]^{-1}\left(h_{i}\right)} & f^{\prime}(1) \leq h_{i}<f^{\prime}(0) \\
0 & h_{i} \geq f^{\prime}(0),\end{cases}
\end{gathered}
$$

where $\left[f^{\prime}\right]^{-1}$ is the inverse of the function $f^{\prime}$. The cutoff for $h_{i} \geq f^{\prime}(0)$ obviously arises only when $f^{\prime}(0)$ is $f$ nite, and is the main difference with the von Neumann case [where Eq. (6) becomes the exponential distribution $p_{i}=e^{-\left(h_{i}+1\right)}$, with $\left.h_{i} \geq-1\right]$. Nevertheless, due to the concavity of $f, p_{i}$ is always a nonincreasing function of $h_{i}\left[p^{\prime}\left(h_{i}\right)=1 / f^{\prime \prime}\left(p_{i}\right)<0\right.$ if $f^{\prime}(1)<h_{i}<f^{\prime}(0)$, and 0 if $\left.h_{i}>f^{\prime}(0)\right]$ that vanishes for $h_{i} \rightarrow \infty \forall f$.

Equations (5) and (6) can be easily derived. As $\operatorname{Tr} \rho h=\operatorname{Tr} \rho_{h} h$, with $\rho_{h}=\sum_{i}\left\langle i_{h}|\rho| i_{h}\right\rangle\left|i_{h}\right\rangle\left\langle i_{h}\right|$, Eq. (2) implies $\bar{S}_{f}(\rho) \leq \bar{S}_{f}\left(\rho_{h}\right)$. The optimum density satisfies then $[\rho, h]=0$, so that $\rho_{h}=\rho$ and $\bar{S}_{f}(\rho)=$ $\sum_{i} f\left(p_{i}\right)-p_{i} h_{i}$. Because of the concavity of $f$, this will have a unique maximum for $p_{i} \in[0,1]$, determined by $f^{\prime}\left(p_{i}\right)=h_{i}$ if $f^{\prime}(1)<h_{i}<f^{\prime}(0)$, or otherwise located at one of the borders, which leads to Eq. (6) [for a nonstandard normalization $\langle I\rangle>1$, an upper cutoff $p_{i}=1$ if $h_{i}<f^{\prime}(1)$ would also apply].
Considering $\bar{S}_{f}(\rho)$ and $S_{f}(\rho)$ at the maximum (5) as functions of $\boldsymbol{\lambda} \equiv\left(\lambda_{0}, \ldots, \lambda_{m}\right)$ and $\overline{\boldsymbol{O}} \equiv\left(\left\langle O_{0}\right\rangle, \ldots,\left\langle O_{m}\right\rangle\right)$, respectively, we obtain the thermodynamic relationships,

$$
\begin{gathered}
\frac{\partial \bar{S}_{f}(\boldsymbol{\lambda})}{\partial \lambda_{\alpha}}=-\left\langle O_{\alpha}\right\rangle, \quad \frac{\partial^{2} \bar{S}_{f}(\boldsymbol{\lambda})}{\partial \lambda_{\alpha} \partial \lambda_{\beta}}=A_{\alpha \beta}, \\
\frac{\partial S_{f}(\overline{\boldsymbol{O}})}{\partial\left\langle O_{\alpha}\right\rangle}=\lambda_{\alpha}, \quad \frac{\partial^{2} S_{f}(\overline{\boldsymbol{O}})}{\partial\left\langle O_{\alpha}\right\rangle\left\langle O_{\beta}\right\rangle}=-\left(A^{-1}\right)_{\alpha \beta}, \\
A_{\alpha \beta}=\sum_{i, j}\left\langle i_{h}\left|O_{\alpha}\right| j_{h}\right\rangle\left\langle j_{h}\left|O_{\beta}\right| i_{h}\right\rangle C_{i j}, \\
C_{i j}=-\delta_{i j} p^{\prime}\left(h_{i}\right)+\left(1-\delta_{i j}\right) \frac{p_{j}-p_{i}}{h_{i}-h_{j}} \geq 0 .
\end{gathered}
$$

Only the second derivatives in (7) and (8) depend explicitly on $f$, through the first term in (10). As $C_{i j} \geq 0, A_{\alpha \alpha} \geq 0$. Moreover, all eigenvalues of $A$ are non-negative, i.e., $A_{\alpha}=$ $\sum_{i, j}\left|\left\langle i_{h}\left|Q_{\alpha}\right| j_{h}\right\rangle^{2}\right| C_{i j}$, with $Q_{\alpha}=\sum_{\beta} U_{\beta \alpha} O_{\beta}$ and $U$ defined by $\left[U^{\text {tr }} A U\right]_{\alpha \beta}=A_{\alpha} \delta_{\alpha \beta}$. Hence, $\bar{S}_{f}$ is a convex function of $\boldsymbol{\lambda}$, whereas $S_{f}$ is a concave function of $\overline{\boldsymbol{O}}$, as in the von Neumann case. If $\left[O_{\alpha}, O_{\beta}\right]=0 \quad \forall \alpha, \beta$, Eq. (9) becomes $A_{\alpha \beta}=$ $-\operatorname{Tr} \rho^{\prime} O_{\alpha} O_{\beta}$, with $\rho^{\prime} \equiv \sum_{i} p^{\prime}\left(h_{i}\right)\left|i_{h}\right\rangle\left\langle i_{h}\right|$ [for $f(p)=$ $-p \ln p, p^{\prime}\left(h_{i}\right)=-p_{i}$ and $\left.\rho^{\prime}=-\rho\right]$.

We will be interested here in functions of the form

$$
f(p)=k\left[p-g_{q}(p)\right],
$$

where $k>0$ and $g_{q}(p)$ is a convex $\left[g_{q}^{\prime \prime}(p)>0\right]$ increasing function satisfying $g_{q}(0)=0, g_{q}(1)=1$, and

$$
\lim _{q \rightarrow \infty} g_{q}\left(p_{i}\right) / g_{q}\left(p_{j}\right)=0 \quad \text { if } p_{i}<p_{j} .
$$

Hence, for sufficiently large $q$ (and finite dimension $n$ ),

$$
S_{f}(\rho)=k\left[1-\operatorname{Tr} g_{q}(\rho)\right] \approx k\left[1-n_{M} g_{q}\left(p_{M}\right)\right],
$$

where $p_{M}$ is the largest eigenvalue of $\rho$ and $n_{M}$ its multiplicity. The density that maximizes $S_{f}(\rho)$ [i.e., minimizes $\left.\operatorname{Tr} g_{q}(\rho)\right]$ subject to a given set of constraints, will then possess, for $q \rightarrow \infty$, the minimum largest eigenvalue $p_{M}$ compatible with the available data, as in this case $n_{M} g_{q}\left(p_{M}\right)$ is minimum. This property may in fact be fulfilled already for finite values of $q$ (i.e., typically $q>q_{c}$ ) in some cases, as will be seen below.

Similarly, maximization of the entropy associated with

$$
\tilde{f}(p)=f(1-p)=k\left[1-p-g_{q}(1-p)\right],
$$

which is also concave and satisfies $\tilde{f}(0)=\tilde{f}(1)=0$, leads to a density which possesses, for $q \rightarrow \infty$, the maximum smallest eigenvalue compatible with the available data. In this limit, $g_{q}\left(1-p_{i}\right) / g_{q}\left(1-p_{j}\right) \rightarrow 0$ if $p_{i}>p_{j}$, so that $S_{\tilde{f}}(\rho) \approx k\left[n-1-n_{m} g_{q}\left(1-p_{m}\right)\right]$ for large $q$, with $p_{m}$ the smallest eigenvalue and $n_{m}$ its multiplicity. This is maximum if $p_{m}$ is maximum.

As a particular example, we have in first term

$$
f(p)=\left(p-p^{q}\right) /(q-1), \quad q>0,
$$


which is concave for $q>0$, approaches $-p \ln p$ for $q \rightarrow$ 1 , and is of the form (11) for $q>1$, satisfying (12). In this case, $S_{f}(\rho)=\left(1-\operatorname{Tr} \rho^{q}\right) /(q-1)$ is the Tsallis entropy, which is $\operatorname{sub}$ (super)additive for $q>1(q<1)$, in agreement with Eq. (3) $\left\{\left[p f^{\prime \prime}(p)\right]^{\prime}=q(1-q) p^{q-2}\right\}$. The $q=2$ case is particularly relevant, since maximization of $S_{f}(\rho)$ becomes equivalent to the minimization of $\operatorname{Tr} \rho^{2}=\left[\sum_{i<j}\left(p_{i}-p_{j}\right)^{2}+1\right] / n$, i.e., to a least squares condition for the probabilities or their differences. For $q=2, S_{f}(\rho)$ also coincides with the information measure of Ref. [8]. Equation (6) becomes, setting $h_{c}=f^{\prime}(0)$,

$$
p_{i}=\left\{\left[1-(q-1) h_{i}\right] / q\right\}^{1 /(q-1)}, \quad-1 \leq h_{i}<h_{c},
$$

and $p_{i}=0$ if $h_{i} \geq h_{c}$, with $h_{c}=\frac{1}{q-1}(\infty)$ if $q>1$ $(q<1)$.

Another example is the exponential function,

$$
f(p)=q^{-1}\left[p-\left(e^{q p}-1\right) /\left(e^{q}-1\right)\right],
$$

which is concave for any $q$, satisfies $f(0)=f(1)=0$, and approaches $\frac{1}{2} p(1-p)$ for $q \rightarrow 0$, i.e., proportional to the Tsallis case $q=2$. For $q>0$, it is of the form (11) and fulfills Eq. (12). Moreover, $f_{-q}(p)=f_{q}(1-$ $p)$. As $\left[p f^{\prime \prime}(p)\right]^{\prime}=e^{q p}(1+q p) q /\left(1-e^{q}\right), S_{f}(\rho)$ is subadditive for $q \geq-1$. Equation (6) becomes

$$
p_{i}=q^{-1} \ln \left[1-\left(e^{q}-1\right) \tilde{h}_{i}\right], \quad-1 \leq \tilde{h}_{i}<0,
$$

and $p_{i}=0$ if $\tilde{h}_{i} \equiv h_{i}-h_{c} \geq 0$, with $h_{c}=\frac{1}{q}-\frac{1}{e^{q}-1}$.

Application.-Let us consider now a bipartite spin $\frac{1}{2}$ system. Starting from the basic unentangled states $|\uparrow \uparrow\rangle,|\uparrow \downarrow\rangle$, $|\downarrow \uparrow\rangle$, and $|\downarrow \downarrow\rangle$, the Bell basis is formed by the fully entangled orthonormal states $\left|\Psi^{ \pm}\right\rangle=(|\uparrow \downarrow\rangle \pm|\downarrow \uparrow\rangle) / \sqrt{2},\left|\Phi^{ \pm}\right\rangle=$ $(|\uparrow \uparrow\rangle \pm|\downarrow \downarrow\rangle) / \sqrt{2}\left(\left|\Psi^{-}\right\rangle\right.$is the singlet while $\left|\Psi^{+}\right\rangle,\left|\Phi^{ \pm}\right\rangle$are spin 1 states with $\langle\boldsymbol{S}\rangle=0$ ). We will consider here Bell constraints [1], i.e., mean values of observables which are diagonal in the Bell basis. Let us first examine the case of Ref. [1], where the available information is the expectation value of the (scaled) Bell-CHSH observable,

$$
B=\left|\Phi^{+}\right\rangle\left\langle\Phi^{+}|-| \Psi^{-}\right\rangle\left\langle\Psi^{-}\right| .
$$

According to Eq. (5), the density $\rho$ that satisfies

$$
\operatorname{Tr} \rho=1, \quad \operatorname{Tr} \rho B=b, \quad|b| \leq 1
$$

and maximizes (1) is of the form

$$
\begin{aligned}
\rho= & p\left(\lambda_{0} I+\lambda_{1} B\right)=p_{0}\left(\left|\Psi^{+}\right\rangle\left\langle\Psi^{+}|+| \Phi^{-}\right\rangle\left\langle\Phi^{-}\right|\right) \\
& +p_{+}\left|\Phi^{+}\right\rangle\left\langle\Phi^{+}\left|+p_{-}\right| \Psi^{-}\right\rangle\left\langle\Psi^{-}\right|,
\end{aligned}
$$

where $p_{0}=p\left(\lambda_{0}\right), p_{ \pm}=p\left(\lambda_{0} \pm \lambda_{1}\right)$. The constraints (17) become just $2 p_{0}+p_{+}+p_{-}=1, p_{+}-p_{-}=b$. We may consider $b \geq 0$, in which case $\lambda_{1} \leq 0$ and $p_{+} \geq$ $p_{0} \geq p_{-}$, since for $b \rightarrow-b, \lambda_{1} \rightarrow-\lambda_{1}$ and $p_{ \pm} \rightarrow p_{\mp}$.

If $f^{\prime}(1)<\lambda_{0} \pm \lambda_{1}<f^{\prime}(0)$, there is no cutoff and the constraints lead to the single equation,

$$
\begin{gathered}
f^{\prime}\left(p_{+}\right)+f^{\prime}\left(p_{+}-b\right)-2 f^{\prime}\left(\frac{1+b}{2}-p_{+}\right)=0, \\
|b|<b_{c},
\end{gathered}
$$

which determines $p_{+}$, and, hence, $p_{-}=p_{+}-b, p_{0}=$ $\frac{1}{2}(1+b)-p_{+}$, for a given $f$. If $f^{\prime}(0)$ is finite, a root of
Eq. (19) for $p_{+} \in\left[b, \frac{1}{2}(1+b)\right]$ will exist only if $|b|<$ $b_{c}$, with $b_{c}$ the root of

$$
f^{\prime}\left(b_{c}\right)+f^{\prime}(0)-2 f^{\prime}\left(\frac{1-b_{c}}{2}\right)=0 .
$$

Equation (20) is equivalent to $f^{\prime}(0)=\lambda_{0}-\lambda_{1}$, and determines the onset of the cutoff for $p_{-}$. It has a single root $b_{c} \in\left[\frac{1}{3}, 1\right]$, with $b_{c} \rightarrow 1$ if $f^{\prime}(0) \rightarrow \infty$. For $b>b_{c}$, $\lambda_{0}-\lambda_{1}>f^{\prime}(0)$, and we obtain the solution

$$
p_{+}=b, \quad p_{-}=0, \quad b_{c} \leq b \leq 1 .
$$

Equations (19) -(21) become apparent from the entropy

$$
S_{f}(\rho)=f\left(p_{+}\right)+f\left(p_{+}-b\right)+2 f\left(\frac{1+b}{2}-p_{+}\right) .
$$

For fixed $b \geq 0$, Eq. (22) is a concave function of $p_{+}$for $p_{+} \in\left[b, \frac{1}{2}(1+b)\right]$, with its maximum located within the interval if $|b|<b_{c}$, being then determined by (19), and at the left border if $b_{c} \leq b \leq 1$, leading to (21). At the maximum, $\lambda_{1}=\partial S_{f}(\rho) / \partial b=f^{\prime}\left(p_{+}\right)-f^{\prime}\left(p_{0}\right)$ in both cases, with $\lambda_{0}=f^{\prime}\left(p_{0}\right)$. Equations (19)-(21) imply that $p_{+}$is an increasing function of $b$ for $b>0$.

For $b \rightarrow 0$, Eq. (19) leads to

$$
p_{+}=\frac{1}{4}\left(1+2 b+\gamma b^{2}\right)+O\left(b^{4}\right),
$$

where $\gamma=-\frac{1}{4} \frac{f^{\prime \prime \prime}(1 / 4)}{f^{\prime \prime}(1 / 4)}<1(>1)$ if $S_{f}$ is sub(super)additive and satisfies Eq. (3). Hence, for $b=0$, we obtain the uniform distribution $p_{+}=p_{-}=p_{0}=\frac{1}{4}$ for any $f$. For $b \rightarrow 1, p_{+} \rightarrow 1$ and $\rho \rightarrow\left|\Phi^{+}\right\rangle\left\langle\Phi^{+}\right|$.

The important question that now arises is whether, for a given $f$, the previous scheme gives fake entanglement. A density $\rho$ diagonal in the Bell basis is unentangled if and only if its largest eigenvalue is not greater than $\frac{1}{2}$ [6]. The density of the form (18) that complies with (17) and possesses the minimum largest eigenvalue corresponds to

$$
\begin{gathered}
p_{+}=\frac{1}{4}(1+b), \quad p_{-}=\frac{1}{4}(1-3 b), \quad 0 \leq b \leq \frac{1}{3}, \\
p_{+}=b, \quad p_{-}=0, \quad \frac{1}{3} \leq b \leq 1, \quad \text { (24) }
\end{gathered}
$$

where $p_{+} \geq p_{0} \geq p_{-}$. Unentangled solutions are then feasible only if $b \leq \frac{1}{2}$. Note also that, for Bell constraints, entanglement is minimized by densities which are diagonal in the Bell basis [1], so that no unentangled density of any form complying with (17) exists for $b>\frac{1}{2}$. It is now seen from (21) that, when $f^{\prime}(0)$ is finite, the maximum entropy density coincides with (24) for $b>b_{c}>\frac{1}{3}$. Hence, as $p_{+}$is an increasing function of $b$, fake entanglement will be avoided for those $f$ for which $b_{c} \leq \frac{1}{2}$.

Particular solutions. - In the von Neumann case, Eq. (19) yields $p_{+}=\frac{1}{4}(1+b)^{2}$, with $b_{c}=1$, in agreement with Ref. [1] and Eq. (23) $(\gamma=1)$. Fake entanglement occurs for $\sqrt{2}-1<b<\frac{1}{2}$.

In the Tsallis case (14), $f^{\prime}(0)$ is finite for $q>1$ and Eq. (20) leads to

$$
b_{c}=\left[1+2^{1-1 /(q-1)}\right]^{-1}, \quad q>1,
$$

which is a decreasing function of $q$ satisfying $b_{c} \leq \frac{1}{2}$ for $q \geq 2$. Hence, fake entanglement will be avoided 
$\forall q \geq 2$. For $q=2$, the solution of Eq. (19) is especially simple, $p_{+}=\frac{1}{4}(1+2 b)$ if $|b|<\frac{1}{2}$ and $p_{+}=b$ if $b \geq \frac{1}{2}$, which is in agreement with (23) $(\gamma=2-q)$ and represents the solution of minimum squares. The onset of entanglement occurs here exactly at $b=b_{c}$.

Although a simple analytic solution of (19) for arbitrary $q$ is not feasible, it is easy to verify that Eq. (24) is obtained for $q \rightarrow \infty \forall b$. In this limit, $b_{c} \rightarrow \frac{1}{3}$, while Eq. (19) yields, for large $q, p_{+} \approx \frac{1}{2}(1+b)\left[1+2^{-1 /(q-1)}\right]^{-1}$, which approaches $\frac{1}{4}(1+b)$ for $q \rightarrow \infty$.

For the exponential function (15), the solution of Eq. (19) is analytic for any $q$ :

$$
\begin{gathered}
p_{+}=\frac{1}{4}(1+2 b)-\frac{1}{2 q} \ln \cosh \left(\frac{1}{2} b q\right), \quad|b|<b_{c}, \\
b_{c}=\frac{1}{3}+\frac{2}{q} \ln \left[\beta_{q}-\frac{e^{-q / 3}}{3 \beta_{q}}\right],
\end{gathered}
$$

with $\beta_{q}=\left[1+\sqrt{1+\left(e^{-q} / 27\right)}\right]^{1 / 3}$ and $p_{+}=b$ for $b \geq b_{c}$. For $q \rightarrow \infty, b_{c} \rightarrow \frac{1}{3}$ and (26) leads immediately to the solution with the minimum largest eigenvalue, Eq. (24). Again, $b_{c}$ is a decreasing function of $q$, with $b_{c}<\frac{1}{2}$ for $q>0$, so that fake entanglement is here avoided $\forall q>0$. For $q \rightarrow 0, b_{c} \rightarrow \frac{1}{2}$ and Eq. (26) reduces to the minimum squares solution. For $b \rightarrow 0$, $p_{+} \approx \frac{1}{4}\left(1+2 b-\frac{1}{4} q b^{2}\right)$, in agreement with (23). Finally, for $q \rightarrow-\infty, b_{c} \rightarrow 1$ and $p_{+} \rightarrow \frac{1}{4}(1+3 b)$, with $p_{-}=p_{0}=\frac{1}{4}(1-b)$, which is the solution with the maximum smallest eigenvalue of $\rho$. This gives fake entanglement for $b \in\left[\frac{1}{3}, \frac{1}{2}\right]$, the maximum interval for maximum entropy densities, as $p_{+}$is in this case maximum.

Inclusion of the dispersion.-If the dispersion of $B$ is also provided [2], through the expectation value of $B^{2}=\left|\Phi^{+}\right\rangle\left\langle\Phi^{+}|+| \Psi^{-}\right\rangle\left\langle\Psi^{-}\right|$, the final maximum entropy density is actually independent of the choice of $f$. In this case $\rho=p\left(\lambda_{0}+\lambda_{1} B+\lambda_{2} B^{2}\right)$ is also of the form (18), with $p_{ \pm}=p\left(\lambda_{0} \pm \lambda_{1}+\lambda_{2}\right)$, $p_{0}=p\left(\lambda_{0}\right)$, which are completely determined by the constraints, i.e., $\quad p_{ \pm}=\frac{1}{2}\left(b_{2} \pm b\right), \quad p_{0}=\frac{1}{2}\left(1-b_{2}\right)$, where $b_{2}=\operatorname{Tr} \rho B^{2}=p_{+}+p_{-}$. The only role played here by maximum entropy is to impose a density of the form (18), which holds for any $f$, and fake entanglement is then always avoided. Note also that, when only $b$ is given, the solution (21) implies minimum dispersion, as $b_{2}=2 p_{-}+b$ is minimum (see also Ref. [2]).

General Bell constraints. - The present arguments are valid for any type of Bell constraints. In this case, densities of minimum entanglement, as measured by the entanglement of formation $E_{F}(\rho)$ [16], are diagonal in the Bell basis [1], and possess the minimum largest eigenvalue $p_{M}$ compatible with the available data if $p_{M}>\frac{1}{2}$. This is so because $E_{F}(\rho)$ is an increasing function of the concurrence $C(\rho)$, which for a system of two qubits reduces to $2 p_{M}-1$ if $p_{M}>\frac{1}{2}$ (and 0 otherwise) when $\rho$ is diagonal in the Bell basis [5,17]. Maximum entropy densities constructed with functions satisfying (11) and (12) will then possess minimum entanglement for $q \rightarrow \infty$, although in some cases this may hold already for finite values of $q$, as seen in the example. For sufficiently large $q$, the entropies $S_{f}(\rho)$ will be essentially decreasing functions of $p_{M}$, being then good entanglement indicators for these densities. This may also be the case in systems of $n$ qubits for special constraints that lead to densities diagonal in a basis of fully entangled states (like the Greenberger-Horne-Zeilinger states used in [18]), where separability is again favored by low values of the largest eigenvalue. Further investigations in this direction are in progress.

In summary, we have examined the use of general nonadditive entropic forms for the inference of quantum density operators. The formalism enables a direct way to infer least biased densities with minimum entanglement for a system of two qubits, and, hence, to avert fake entanglement, when the information consists of any set of Bell constraints. It also provides a general framework for the description of the thermodynamic aspects of entanglement, as well as a more deep foundation of the success that nonadditive entropies such as that of Tsallis may encounter in this type of problems.

N.C. and R. R. acknowledge support from CONICET and CIC, respectively, of Argentina.

[1] R. Horodecki, M. Horodecki, and P. Horodecki, Phys. Rev. A 59, 1799 (1999).

[2] A. K. Rajagopal, Phys. Rev. A 60, 4338 (1999).

[3] S. Abe and A. K. Rajagopal, Phys. Rev. A 60, 3461 (1999).

[4] C. Tsallis, S. Lloyd, and M. Baranger, Phys. Rev. A 63, 042104 (2001).

[5] F. Giraldi and P. Grigolini, Phys. Rev. A 64, 032310 (2001).

[6] R. Horodecki and M. Horodecki, Phys. Rev. A 54, 1838 (1996).

[7] N. J. Cerf and C. Adami, Phys. Rev. Lett. 79, 5194 (1997).

[8] C. Brukner and A. Zeilinger, Phys. Rev. Lett. 83, 3354 (1999).

[9] A. K. Ekert, Phys. Rev. Lett. 67, 661 (1991).

[10] C. H. Bennett et al., Phys. Rev. Lett. 70, 1895 (1993).

[11] C. Beck and F. Schlögl, Thermodynamics of Chaotic Systems: An Introduction (Cambridge University, Cambridge, England, 1993).

[12] C. Tsallis, J. Stat. Phys. 52, 479 (1988).

[13] C. Tsallis et al., Phys. Rev. Lett. 75, 3589 (1995); C. Tsallis and D. J. Bukman, Phys. Rev. E 54, R2197 (1996); M. L. Lyra and C. Tsallis, Phys. Rev. Lett. 80, 53 (1998).

[14] A. Wehrl, Rev. Mod. Phys. 50, 221 (1978).

[15] R. Rossignoli and N. Canosa, Phys. Lett. A 264, 148 (1999).

[16] C. H. Bennett et al., Phys. Rev. A 54, 3824 (1996).

[17] W. K. Wootters, Phys. Rev. Lett. 80, 2245 (1998).

[18] W. Dür, J. I. Cirac, and R. Tarrach, Phys. Rev. Lett. 83, 3562 (1999). 